\title{
Utilization of the dynamic laser scanning technology for monitoring, locating and classification of the city trees.
}

\begin{abstract}
One of the significant issues related to the city trees is the transmission overhead cables damages caused by the trees growth that would sometime generate power disconnections. Currently the city progress monitoring technologies have three major challenges. Firstly, the spatial resolution of the collected data is critical factor in all monitoring technologies that would reflect the utilization of the extracted data and physical consistency with the ground features. Secondly, the temporal resolution where in all progress monitoring or change detection technologies the duration and time coverage is a basic for validating extracted features. Finally, the human resources and the operational capital is also primary cost when monitoring city trees is needed on a regular basis. Therefore, the research is intended to verify the feasibility of allocating the trees that overheads the electrical cables especially where the transmission overhead cables passing the forests. The area of interest is extended along the highways where most of the overhead cables are placed. The research is utilizing the dynamic laser scanning system for progress monitoring and spatially locating the well recognized and accessed city trees.
\end{abstract}

Keyword: GIS; Smart cities; Mobile laser scanning; City monitoring. 